Post-print of: Food Chemistry $183.78-82(2015)$

\title{
Assessment of white grape pomace from winemaking as source of bioactive compounds, and its antiproliferative activity
}

\author{
M. José Jara-Palacios ${ }^{\mathrm{a}}$, Dolores Hernanz ${ }^{\mathrm{b}}$, Tania Cifuentes-Gomez ${ }^{\mathrm{c}}$, M. Luisa Escudero-Gilete ${ }^{\mathrm{a}}$, \\ Francisco J. Heredia $^{a, *}$, Jeremy P.E. Spencer ${ }^{c}$ \\ ${ }^{a}$ Food Colour and Quality Laboratory, Department of Nutrition and Food Science, Facultad de Farmacia, Universidad de Sevilla, 41012 Sevilla, Spain \\ ${ }^{\mathrm{b}}$ Department of Analytical Chemistry, Facultad de Farmacia, Universidad de Sevilla, 41012 Sevilla, Spain \\ ${ }^{\mathrm{c}}$ Molecular Nutrition Group, Department of Food and Nutritional Sciences, School of Chemistry, Food and Pharmacy, University of Reading, Reading RG6 6AP, United Kingdom
}

Keywords:

Caco-2

Colorectal cancer

Grape pomace

Phenolic compounds

\begin{abstract}
A B S T R A C T
The antiproliferative effects of a purified white grape pomace extract (PWGPE), as well as of some phenolic standards on colon cancer cells were examined. The phenolic composition of the PWGPE was determined by rapid resolution liquid chromatography/mass spectrometry (RRLC/MS). The PWGPE had 92.6, 43.3 and $6.01 \mathrm{mg} / \mathrm{g}$ of flavanols, flavonols and phenolic acids, respectively and, along with pure catechin, epicatechin, quercetin and gallic acid, they were all found capable of inhibiting cellular proliferation. PWGPE $(100 \mu \mathrm{g} / \mathrm{ml})$ inhibited the proliferation of cells by $52.1 \%$ at $48 \mathrm{~h}$, whilst catechin, epicatechin, quercetin and gallic acid $(60 \mu \mathrm{g} / \mathrm{ml})$ inhibited growth by $65.2 \%, 62.2 \%, 81.0 \%$ and $71.0 \%$, respectively, at $72 \mathrm{~h}$. The PWGPE is an interesting source of phenolic compounds with antiproliferative properties, that could be of interest in the food and pharmaceutical industries.
\end{abstract}

(c) 2015 Elsevier Ltd. All rights reserved.

\section{Introduction}

Wine seems to be beneficial to health and, therefore, a moderate and regular consumption of wine is recommended. During winemaking, the polyphenols of the grape are transferred to the wine, but a high proportion still remains in the solid winemaking by-products. Grape pomace, consisting of the waste seeds, skins and stems resulting from the winemaking process, has been postulated to be a relatively good source of dietary bioactive compounds, such as polyphenols (Anastasiadi, Pratsinis, Kletsas, Skaltsounis, \& Haroutounian, 2010; Rodríguez Montealegre, Romero Peces, Chacón Vozmediano, Martínez Gascueña, \& García Romero, 2006), with recent data reporting its antioxidant, antiradical, antimicrobial, anti-inflammation and anticancer activities and its cardioprotective action (Rodríguez-Rodríguez et al., 2012; Sagdic et al., 2011; Xia, Deng, Guo, \& Li, 2010). Therefore, the consumption of polyphenols has been associated with reduced risk of chronic diseases, including cardiovascular and neurodegenerative diseases, and cancer, e.g., colon cancer (Vauzour, Rodriguez-Mateos, Corona, Oruna-Concha, \& Spencer, 2010).

The importance of polyphenols makes the study of byproducts of interest for the food, nutraceutical, pharmaceutical and chemical industries. In recent years, there had been a growing interest in the use of byproducts rich in polyphenols emanating, from the winemaking industry, to produce extracts and novel products for the maintenance of human health (Kulkarni, De Santos, Kattamuri, Rossi, \& Brewer, 2011; Perumalla \& Hettiarachchy, 2011). As such, the extraction of polyphenols from waste material deriving from traditional industrial practices represents an attractive, sustainable and cost effective source of these high-value biological bioactives, which could be incorporated into foods. Due to the increasing demand for nutraceutical and antioxidant compounds, the study of grape pomace may be useful for industrial purposes.

Colorectal cancer is the third most prevalent cancer worldwide (Chan \& Giovannucci, 2010) and there is intense interest in the development of novel chemopreventive products and/or the production of anti-cancer agents from novel, industrial waste-stream sources. Several studies have indicated the anti-cancer potential of polyphenolic compounds, and in particular their ability to inhibit the proliferation of cancer cells via their effects on the cell-cycle and/or apoptosis (Lee et al., 2006; Ramos, Rodríguez-Ramiro, Martín, Goya, \& Bravo, 2011; Vu et al., 2012), and have suggested their use as novel dietary chemopreventive agents (Araújo, Gonçalves, \& Martel, 2011). For example, the antiproliferative effects of polyphenolic extracts from olive oil (Corona et al., 2007), red wine (Gómez-Alonso et al., 2012), tomato (Saunders, 2009), araçá (Medina et al., 2011), raspberry (Coates et al., 2007), 
and cranberry (Vu et al., 2012) have been reported. However, to date there are no previous data on the actions of grape pomace extracts, although their potential to protect against oxidative stress has been shown in Caenorhabditis elegans (Jara-Palacios et al., 2013). These phenolic extracts are a relatively rich source of bioactive compounds, which could be exploited in the food and pharmaceutical industries.

The current study we aimed to address this by investigating the potential anti-cancer effects of a purified white grape pomace extract (PWGPE), rich in phenolic compounds, on colon adenocarcinoma cell growth, in relation to the individual effects of pure polyphenols relevant to PWGPE, notably epicatechin, catechin, quercetin and gallic acid.

\section{Materials and methods}

\subsection{Reagents and standards}

Methanol, $n$-hexane and hydrochloric acid were purchased from Labscan (Dublin, Ireland). Formic acid, HPLC-grade acetonitrile, sodium carbonate, and Folin-Ciocalteau reagent were obtained from Panreac (Barcelona, Spain). Gallic acid, protocatechuic acid, (+)-catechin, (-)-epicatechin, quercetin, kaempferol, quercetin-3-O-rutinoside (rutin), ferulic acid, caffeic acid, $p$-coumaric acid, and phosphate buffered saline (PBS) were purchased from Sigma-Aldrich (Madrid, Spain). Quercetin-3-O-glucoside and kaempferol-3-O-glucoside were obtained from Extrasynthese (Genay, France). Alamar Blue was from Abd Serotec (Kidlington, UK). Fehling's reagents, dimethyl sulfoxide (DMSO), sulforhodamine B-based assay kit and all other reagents used for assay with cells were obtained from Sigma Aldrich (UK).

The stock solutions of phenolic standards were prepared in water at concentration of $100 \mathrm{mg} / \mathrm{L}$. The corresponding dilutions were made up from the stock solutions to assess the antiproliferative effects.

\subsection{Extraction of phenolic compounds from grape pomace}

Grape pomace of the variety Zalema, D.O. "Condado de Huelva" (Spain), collected after winemaking, was supplied by "Vinícola del Condado" winery (Bollullos Par del Condado, Spain). Grape pomace was immediately frozen at $-20^{\circ} \mathrm{C}$, freezedried for $48 \mathrm{~h}$ and finally ground to a fine powder. The freezedried grape pomace ( $50 \mathrm{~g}$ ) was extracted with $75 \%$ methanol as extraction solvent. The mixture was sonicated for $1 \mathrm{~h}$ followed by further constant shaking for $12 \mathrm{~h}$ (VWR Incubating mini-shaker), centrifuged at $4190 \times g$ for $15 \mathrm{~min}$ and finally the supernatant was collected. The remaining residue was subjected to a further two extractions and the supernatants were combined to obtain the phenolic extract. This methanolic phenolic extract was dried under vacuum and the aqueous extract was washed with $n$-hexane. The resulting extract was passed through a C18 column $(10 \times 5 \mathrm{~cm})$ to remove sugars, which was achieved by their elution, following addition of $100 \mathrm{ml}$ of acidified water $(\mathrm{pH} 2 ; \mathrm{HCl})$, followed by $300 \mathrm{ml}$ of Milli- ${ }^{\circledR}$ water at a constant rate $(0.5 \mathrm{ml} /$ min), using a diaphragm-metering pump (STEPDOS, Scientific Laboratory Suppliers). The Fehling test was carried out to ascertain the presence of sugars in the water extracts, as described previously (Eid, Al-Awadi, Vauzour, Oruna-Concha, \& Spencer, 2013). Following the removal of sugars, the elution of phenolic compounds was achieved by addition of $400 \mathrm{ml}$ of methanol. This methanolic extract was concentrated to dryness, using a rotatory vacuum evaporator at $40{ }^{\circ} \mathrm{C}$, and freeze-dried, yielding the PWGPE.

\subsection{Determination of individual phenolic compounds: RRLC/MS analysis}

Chromatographic analysis was carried out as previously described (Jara-Palacios et al., 2014). An Agilent 1260 chromatograph (Agilent Technologies, Palo Alto, CA, USA), equipped with a diode-array detector, and an API 3200 Qtrap (Applied Biosystems, Darmstadt, Germany), equipped with an ESI source, and a triple-quadrupole ion trap mass analyser were used. Phenolic compounds were identified by comparing their retention time, UV-vis spectra, and mass spectral data with authentic standards where available. The compounds were quantified using peak area data of resolved peaks at 280, 320 and $370 \mathrm{~nm}$, for flavanols, phenolic acids and flavonols, respectively. Procyanidins were quantified, using catechin as the standard, whilst caftaric, fertaric and coutaric acids were quantified using caffeic, ferulic and $p$-coumaric acids, respectively. Quercetin and isorhamnetin derivatives were quantified as quercetin, and kaempferol derivates as kaempferol. Detailed retention times, UV-vis data and mass spectrometric data were shown previously in another paper (Jara-Palacios et al., 2014). The samples were analyzed in triplicate, and the results were expressed as $\mathrm{mg}$ of phenolic compound per $\mathrm{g}$ of PWGPE.

\subsection{Determination of total phenolic content}

The total phenolic content (TPC) was determined by the FolinCiocalteu spectrophotometric method, as described previously (Jara-Palacios et al., 2014). Analyses were conducted in triplicate and gallic acid was employed as a calibration standard. Results were expressed as $\mathrm{mg}$ of gallic acid per $\mathrm{g}$ of PWGPE.

\subsection{Cell culture}

\subsubsection{General}

Human colon adenocarcinoma cells (Caco-2, ECACC Salisbury, Wiltshire, UK) were cultured in Dulbecco's modified Eagle's medium (DMEM), supplemented with $20 \%$ heat-inactivated bovine serum, $2 \mathrm{mM}$ L-glutamine, $1 \%$ non-essential amino acids, $100 \mathrm{U} /$ $\mathrm{ml}$ of penicillin, and $100 \mu \mathrm{g} / \mathrm{ml}$ of streptomycin. Cells were seeded in 24 -well plates at low confluence $\left(2.5 \times 10^{4}\right.$ per well $)$ and then exposed to the PWGPE $(10,25,50$ and $100 \mu \mathrm{g} / \mathrm{ml})$, catechin (30 and $60 \mu \mathrm{g} / \mathrm{ml}$ ), epicatechin (30 and $60 \mu \mathrm{g} / \mathrm{ml}$ ), quercetin (30 and $60 \mu \mathrm{g} / \mathrm{ml}$ ), gallic acid (30 and $60 \mu \mathrm{g} / \mathrm{ml}$ ), or vehicle (DMSO 1\%), $8 \mathrm{~h}$ after seeding.

\subsubsection{Cellular proliferation and viability}

The ability of the PWGPE to inhibit cellular proliferation was tested, using the sulforhodamine B (SRB) assay. Cells were harvested following 24,48 and $72 \mathrm{~h}$ in culture and treated by the addition of $125 \mu \mathrm{l}$ of ice-cold TCA ( $4{ }^{\circ} \mathrm{C} ; 1 \mathrm{~h}$ ). After fixing, media were removed, cells were washed and total biomass was determined using SRB ( $250 \mu \mathrm{l}$ of $0.4 \% \mathrm{SRB}$; $30 \mathrm{~min})$. Unincorporated dye was removed by washing with $1 \%$ acetic acid, whilst cell incorporated dye was solubilised using Tris-base $(10 \mathrm{mM})$. Dye incorporation, reflecting cell biomass, was measured at $492 \mathrm{~nm}$, using a GENios microplate reader (TECAN, Reading, UK).

Viability was assessed after $24 \mathrm{~h}$ of treatment with PWGPE at different concentrations by the Alamar blue assay, as described previously (Vauzour, Vafeiadou, Rice-Evans, Williams, \& Spencer, 2007). Briefly, Alamar Blue solution ( $10 \% \mathrm{v} / \mathrm{v})$ was added to each well, and plates were incubated for $3 \mathrm{~h}$, prior to fluorescence being measured at $540 \mathrm{~nm}$ excitation and $612 \mathrm{~nm}$ emission on the GENios microplate reader. 


\subsection{Statistical analysis}

For the statistical treatment of the data, the Statistica v.8.0 software was used. One-way analysis of variance (ANOVA) was employed to establish if antiproliferative effects differed significantly between (a) the PWGPE and the phenolic standards and (b) those with the vehicle (DMSO 1\%).

\section{Results and discussion}

\subsection{Phenolic composition}

The total phenolic content of the PWGPE was $365 \mathrm{mg} / \mathrm{g}$ (Table 1). 11 flavanols, 9 flavonols and 5 phenolic acids were identified and quantified by RRLC/MS, with flavanols being the major compounds detected accounting for $65 \%$ of total phenolic compounds $(92.6 \mathrm{mg}$ flavanols/g PWGPE), followed by flavonols ( $43.3 \mathrm{mg} / \mathrm{g}$ PWGPE, $31 \%$ ) and phenolic acids (6.01 mg/g PWGPE, 4\%) (Table 1 ). The most abundant flavanol was procyanidin B1 (15.5 mg/g PWGPE), followed by catechin, procyanidin $\mathrm{B} 2-3-0$-gallate, and procyanidin tetramer 1 (12.2, 11.8 and $10.8 \mathrm{mg} / \mathrm{g}$ PWGPE, respectively). The main flavonols were quercetin-3-O-glucoside and quercetin-3-O-glucuronide (16.9 and $15.8 \mathrm{mg} / \mathrm{g}$ PWGPE, respectively). The most abundant phenolic acid was gallic acid ( $3.03 \mathrm{mg} / \mathrm{g}$ PWGPE). These phenolic compounds were found in other extracts from Zalema grape pomace, but in different amounts (Jara-Palacios et al., 2013, 2014), which may be due to the extraction process.

\subsection{Effect on cell proliferation}

PWGPE, at different concentrations $(10,25,50$ and $100 \mu \mathrm{g} / \mathrm{ml})$, induced dose-dependent inhibition of cancer cell proliferation

Table 1

Concentrations of phenolic compounds identified by RRLC/MS and TPC of PWGPE.

\begin{tabular}{lc}
\hline Phenolic compound & $\mathrm{mg} / \mathrm{g}$ PWGPE \\
\hline Flavanols & \\
Catechin & $12.2 \pm 0.07$ \\
Epicatechin & $6.34 \pm 0.02$ \\
Procyanidin B1 & $15.5 \pm 0.00$ \\
Procyanidin B2 & $4.94 \pm 0.80$ \\
Procyanidin B3 & $4.35 \pm 0.10$ \\
Galloylated procyanidin & $7.97 \pm 0.00$ \\
Procyanidin B2-3-O-gallate & $11.8 \pm 0.29$ \\
Procyanidin trimer 1 & $5.65 \pm 0.35$ \\
Procyanidin trimer 2 & $7.27 \pm 0.27$ \\
Procyanidin tetramer 1 & $10.8 \pm 0.10$ \\
Procyanidin tetramer 2 & $5.74 \pm 0.02$ \\
Flavonols & \\
Quercetin (Q) & $2.87 \pm 0.00$ \\
Kaempferol (K) & $0.15 \pm 0.00$ \\
Q-3-O-rutinoside & $0.69 \pm 0.00$ \\
Q-3-O-glucuronide & $15.8 \pm 0.01$ \\
Q-3-O-galactoside & $2.12 \pm 0.02$ \\
Q-3-O-glucoside & $16.9 \pm 0.07$ \\
K-3-O-glucuronide & $0.31 \pm 0.00$ \\
K-3-O-glucoside & $3.67 \pm 1.73$ \\
I-3-O-glucoside & $0.70 \pm 0.01$ \\
Phenolic acids & \\
Gallic acid & $3.03 \pm 0.13$ \\
Protocatechuic acid & $0.94 \pm 0.13$ \\
Caftaric acid & $0.88 \pm 0.00$ \\
Caffeic acid & $0.63 \pm 0.01$ \\
p-Coumaric acid & $0.51 \pm 0.01$ \\
Total phenolic compounds ${ }^{\mathrm{a}}$ & $142 \pm 1.41$ \\
Total phenolic content ${ }^{\mathrm{B}}$ & $365 \pm 3.35$ \\
\hline isorhamnetin. Each value & \\
\hline
\end{tabular}

I, isorhamnetin. Each value represents mean $(n=9) \pm \mathrm{SD}$.

a Sum of individual phenolic compounds.

b TPC determined by Folin Ciocalteu assay. following 24, 48 and $72 \mathrm{~h}$ of exposure (Fig. 1). A significant increase $(p<0.05)$ in growth inhibition treated cells was observed after all treatments, relative to vehicle, with the exception of PWGPE at $10 \mu \mathrm{g} / \mathrm{ml}$ and $72 \mathrm{~h}$ of exposure $(p>0.05)$, whilst the $100 \mu \mathrm{g} / \mathrm{ml}$ dose for $48 \mathrm{~h}$ induced the greatest degree of inhibition of proliferation (52\% growth inhibition). Our data agree with previous cell data indicating that polyphenols are capable of inhibiting the proliferation of colon adenocarcinoma cells in a dose-dependent manner. Notably, the polyphenol rich foods, e.g., olive oil $(100 \mu \mathrm{g} / \mathrm{ml})$, red wine $(100 \mu \mathrm{g} / \mathrm{ml})$ and a tomato extract $(2.5 \mathrm{mg} / \mathrm{ml})$ inhibited cancer cell growth over a similar time frame (Corona et al., 2007; Gómez-Alonso et al., 2012; Saunders, 2009).

Previous studies have indicated that such activity may be mediated via the potential of polyphenols and their metabolites to induce cell cycle blockage in the $\mathrm{G} 2 / \mathrm{M}$ phase in adenocarcinoma cells, perhaps through their effects on COX-2 activity (Shan, Wang, \& Li, 2009; Yasui, Kim, Oyama, \& Tanaka, 2009). On the other hand, these antiproliferative effects may be mediated, in part, by their direct cytotoxic action (Gómez-Alonso et al., 2012). With respect to the latter, our data suggest that cell viability at $24 \mathrm{~h}$ was significantly $(p<0.05)$ reduced at higher exposure concentrations (Fig. 2). Our data agree with previous observations that a polyphenol-rich red wine and araçá fruit extracts also reduce cell viability (Gómez-Alonso et al., 2012; Medina et al., 2011).

The assayed concentrations of polyphenols $(10-100 \mu \mathrm{g} / \mathrm{ml})$ in the culture medium could be associated with those present in some food sources, such as white wine, cocoa, black currant, or tea (Lamuela-Raventós, Romero-Pérez, Andrés-Lacueva, \& Tornero, 2005; Recamales, Sayago, González-Miret, \& Hernanz, 2006; Wang, Lu, Miao, Xie, \& Yang, 2008; Williamson \& Clifford, 2010). These amounts of polyphenols have shown antiproliferative effects in the cells; however, human health effects of these compounds depend on their bioavailability. A previous study suggests that the phenolic composition of a food source could affect the microbiota and its catabolic activity, inducing changes that could in turn affect the bioavailability and potential bioactivity of polyphenols producing metabolites which might be responsible for observed health effects in vivo (Cueva et al., 2013). Therefore, although in vitro assays provide information on bioactivity of polyphenols, it is essential to know their bioavailability by in vivo studies, which would be interesting to look at in future.

In order to elaborate the mediating bioactive components of the extract used, individual phenolic compounds, gallic acid, catechin, quercetin and epicatechin were also tested for their effects on cancer cell growth (Fig. 3). A significant inhibition $(p<0.05)$ of proliferation was observed for all compounds, relative to vehicle-

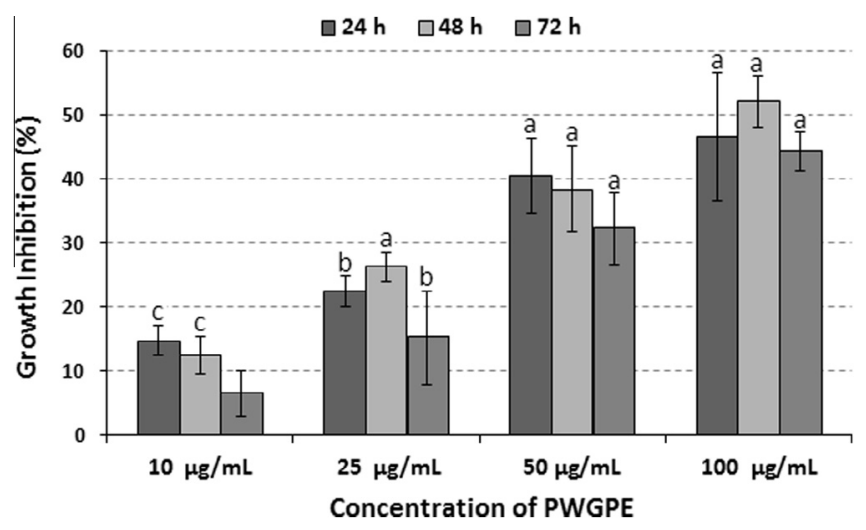

Fig. 1. Growth inhibition induced by treatment with PWGPE at different concentrations. Caco- 2 cells were exposed to the treatment for 24,48 and $72 \mathrm{~h}$ before SRB assay was conducted. Each value represents mean $(n=12) \pm \mathrm{SD} . \mathrm{a}=p<0.001$; $\mathrm{b}=p<0.01 ; \mathrm{c}=p<0.05$. 


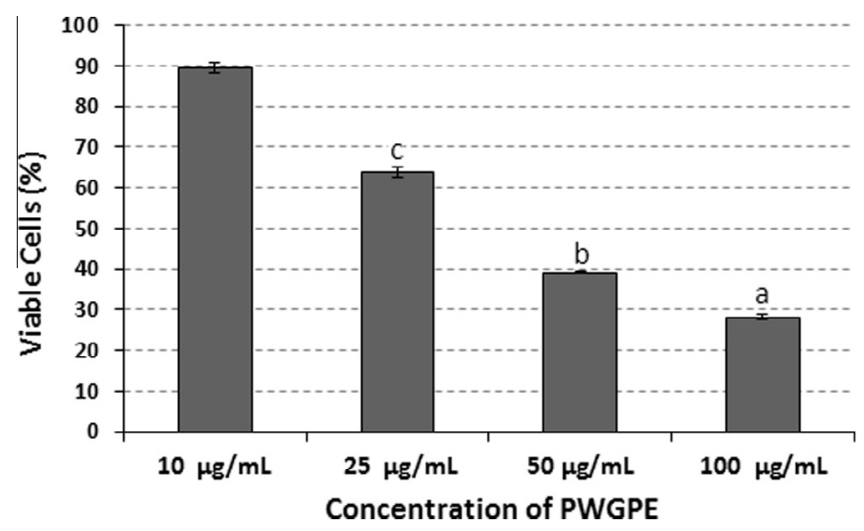

Fig. 2. Viable cells determined after treatment for $24 \mathrm{~h}$ with different concentrations of PWGPE. Caco-2 cells were exposed to the treatment for $24 \mathrm{~h}$ before Alamar blue assay was conducted. Each value represents mean $(n=12) \pm \operatorname{SD} . \mathrm{a}=p<0.001$; $\mathrm{b}=p<0.01 ; \mathrm{c}=p<0.05$.

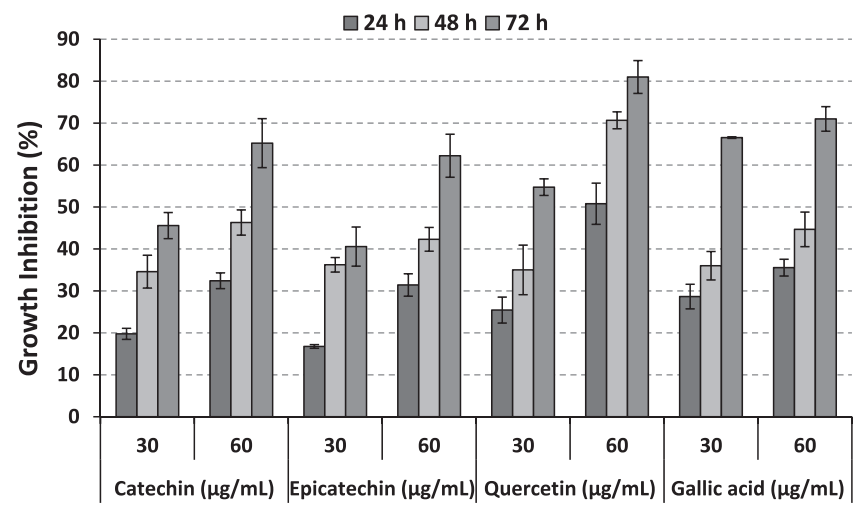

Fig. 3. Growth inhibition induced by treatment with catechin, epicatechin, quercetin and gallic acid at different concentrations. Caco-2 cells were exposed to the treatment for 24,48 and $72 \mathrm{~h}$ before SRB assay was conducted. Each value represents mean $(n=9) \pm \operatorname{SD}$.

treated cells, ranging from $17 \%$ to $81 \%$ for epicatechin $(30 \mu \mathrm{g} / \mathrm{ml})$ and quercetin $(60 \mu \mathrm{g} / \mathrm{ml})$, respectively (Fig. 3). Indeed, the antiproliferative effects of the PWGPE were comparable to effects of catechin, epicatechin and gallic acid at similar concentrations to that found in the extract $(p>0.05)$. Although the precise concentrations of catechin, epicatechin, gallic acid and quercetin varied in the extract (12.2, 6.3, 3.0 and $2.9 \mathrm{mg} / \mathrm{g}$ PWGPE, respectively), as reported previously (Araújo et al., 2011), a combination of polyphenols may target overlapping and complementary phases of the carcinogenic process, thus increasing the efficacy and potency of its chemopreventive effects. Previous data support this, with a variety of phenolic compounds previously described as anticancer compounds with respect to colorectal cancer (Araújo et al., 2011; Ramos et al., 2011; Salucci, Stivala, Maiani, Bugianesi, \& Vannini, 2002).

\section{Conclusions}

In summary, a PWGPE obtained from the winemaking process (Zalema white grape variety) is capable of inhibiting adenocarcinoma cell proliferation by a combination of antiproliferative activity and via a direct initiation of cell death. Further studies are required to elucidate the specific components present in PWGPE which are responsible for these effects and the mechanisms involved, although our data support the conclusion that major polyphenols present in the extract, such as catechin and quercetin, may be the mediating components. This study has described the inhibitory nature of dietary polyphenolic compounds on colon cancer cell growth, but there is no clear evidence that consumption of polyphenols prevents cancer; therefore future work is necessary to establish this.

\section{Acknowledgements}

This work was partially supported by "V Plan Propio de Investigación" of Universidad de Sevilla. The authors acknowledge the collaboration of "Consejo Regulador D.O. Condado de Huelva" and assistance of the technical staff of Biology Service (SGI, Universidad de Sevilla). M.J. Jara-Palacios holds a predoctoral Research Grant (FPU) from the Spanish Ministry of Education.

\section{References}

Anastasiadi, M., Pratsinis, H., Kletsas, D., Skaltsounis, A. L., \& Haroutounian, S. A. (2010). Bioactive non-coloured polyphenols content of grapes, wines and vinification by-products. Evaluation of the antioxidant activities of their extracts. Food Research International, 43, 805-813.

Araújo, J. R., Gonçalves, P., \& Martel, F. (2011). Chemopreventive effect of dietary polyphenols in colorectal cancer cell lines. Nutrition Research, 31, 77-87.

Chan, A. T., \& Giovannucci, E. L. (2010). Primary prevention of colorectal cancer. Gastroenterology, 138, 2029-2043.

Coates, E. M., Popa, G., Gill, C. I., McCann, M. J., McDougall, G. J., Stewart, D., et al. (2007). Colon-available raspberry polyphenols exhibit anti-cancer effects on in vitro models of colon cancer. Journal of Carcinogenesis, 6, 1-10.

Corona, G., Deiana, M., Incani, A., Vauzour, D., Dessì, M. A., \& Spencer, J. P. (2007) Inhibition of p38/CREB phosphorylation and COX-2 expression by olive oil polyphenols underlies their anti-proliferative effects. Biochemical and Biophysical Research Communications, 362, 606-611.

Cueva, C., Sánchez-Patán, F., Monagas, M., Walton, G. E., Gibson, G. R., MartínÁlvarez, P. J., et al. (2013). In vitro fermentation of grape seed flavan-3-ol fractions by human faecal microbiota: Changes in microbial groups and phenolic metabolites. FEMS Microbiology Ecology, 83, 792-805.

Eid, N. M. S., Al-Awadi, B., Vauzour, D., Oruna-Concha, M. J., \& Spencer, J. P. E. (2013) The effect of cultivar type and ripening on the polyphenol content of date palm fruit. Journal of Agricultural and Food Chemistry, 61, 2453-2460.

Gómez-Alonso, S., Collins, V. J., Vauzour, D. Rodríguez-Mateos, A Corona, G. \& Spencer, J. P. E. (2012). Inhibition of colon adenocarcinoma cell proliferation by flavonols is linked to a G2/M cell cycle block and reduction in cyclin D1 expression. Food Chemistry, 130, 493-500.

Jara-Palacios, M. J., González-Manzano, S., Escudero-Gilete, M. L., Hernanz, D., Duen as, M., González-Paramás, A. M., et al. (2013). Study of Zalema grape pomace: Phenolic composition and biological effects in Caenorhabditis elegans. Journal of Agricultural and Food Chemistry, 61, 5114-5121.

Jara-Palacios, M. J., Hernanz, D., González-Manzano, S., Santos-Buelga, C., EscuderoGilete, M. L., \& Heredia, F. J. (2014). Detailed phenolic composition of white grape by-products by RRLC/MS and measurement of the antioxidant activity. Talanta, 125, 51-57.

Kulkarni, S., De Santos, F. A., Kattamuri, S., Rossi, S. J., \& Brewer, M. S. (2011). Effect of grape seed extract on oxidative, color and sensory stability of a pre-cooked, frozen, re-heated beef sausage model system. Meat Science, 88, 139-144.

Lamuela-Raventós, R. M., Romero-Pérez, A. I., Andrés-Lacueva, C., \& Tornero, A. (2005). Review: Health effects of cocoa flavonoids. Food Science and Technology International, 11, 159-176.

Lee, S. Y. H., Munerol, B., Pollard, S., Youdim, K. A., Pannala, A. S., Kuhnle, G. G., et al. (2006). The reaction of flavanols with nitrous acid protects against Nnitrosamine formation and leads to the formation of nitroso derivatives which inhibit cancer cell growth. Free Radical Biology and Medicine, 40, 323-334.

Medina, A. L., Haas, L. I. R., Chaves, F. C., Salvador, M., Zambiazi, R. C., Silva, W. P., et al. (2011). Araçá (Psidium cattleianum Sabine) fruit extracts with antioxidant and antimicrobial activities and antiproliferative effect on human cancer cells. Food Chemistry, 128, 916-922.

Perumalla, A. V. S., \& Hettiarachchy, N. S. (2011). Green tea and grape seed extractspotential applications in food safety and quality. Food Research International, 44, 827-839.

Ramos, S., Rodríguez-Ramiro, I., Martín, M. A., Goya, L., \& Bravo, L. (2011). Dietary flavanols exert different effects on antioxidant defenses and apoptosis/ proliferation in Caco-2 and SW480 colon cancer cells. Toxicology in Vitro, 25, 1771-1781.

Recamales, A. F., Sayago, A., González-Miret, M. L., \& Hernanz, D. (2006). The effect of time and storage conditions on the phenolic composition and colour of white wine. Food Research International, 39, 220-229.

Rodríguez Montealegre, R., Romero Peces, R., Chacón Vozmediano, J. L., Martínez Gascuen a, J., \& García Romero, E. (2006). Phenolic compounds in skins and seeds of ten grape Vitis vinifera varieties grown in a warm climate. Journal of Food Composition and Analysis, 2006(19), 687-693.

Rodríguez-Rodríguez, R., Justo, M. L., Claro, C. M., Vila, E., Parrado, J., Herrera, M. D., et al. (2012). Endothelium dependent vasodilator and antioxidant properties of 
a novel enzymatic extract of grape pomace from wine industrial waste. Food Chemistry, 135, 1044-1051.

Sagdic, O., Ozturk, I., Ozkan, G., Yetim, H., Ekici, L., \& Yilmaz, M. T. (2011). RP-HPLC$\mathrm{DAD}$ analysis of phenolic compounds in pomace extracts from five grape cultivars: Evaluation of their antioxidant, antiradical and antifungal activities in orange and apple juices. Food Chemistry, 126, 1749-1758.

Salucci, M., Stivala, L. A., Maiani, G., Bugianesi, R., \& Vannini, V. (2002). Flavonoids uptake and their effect on cell cycle of human colon adenocarcinoma cells (Caco2). British Journal of Cancer, 86, 1645-1651.

Saunders, C. (2009). The anti-proliferative effect of different tomato varieties on the human colon adenocarcinoma cells. Bioscience Horizons, 2, 172-179.

Shan, B. E., Wang, M. X. \& Li, R. G. (2009). Quercetin inhibit human sw480 colon cancer growth in association with inhibition of cyclin d1 and surviving expression through Wnt/ $\beta$-catenin signaling pathway. Cancer Investigation, 27 , 604-612.

Vauzour, D., Vafeiadou, K., Rice-Evans, C., Williams, R. J., \& Spencer, J. P. E. (2007). Activation of pro-survival Akt and ERK1/2 signalling pathways underlie the anti-apoptotic effects of flavanones in cortical neurons. Journal of Neurochemistry, 103, 1355-1367.
Vauzour, D., Rodriguez-Mateos, A., Corona, G., Oruna-Concha, M. J., \& Spencer, J. P. E. (2010). Polyphenols and human health: Prevention of disease and mechanisms of action. Nutrients, 2, 1106-1131.

Vu, K. D., Carlettini, H., Bouvet, J., Côté, J., Doyon, G., Sylvain, J. F., et al. (2012). Effect of different cranberry extracts and juices during cranberry juice processing on the antiproliferative activity against two colon cancer cell lines. Food Chemistry, 132, 959-967.

Wang, D., Lu, J., Miao, A., Xie, Z., \& Yang, D. (2008). HPLC-DAD-ESIMS/MS analysis of polyphenols and purine alkaloids in leaves of 22 tea cultivars in China. Journal of Food and Composition and Analysis, 21, 361-369.

Williamson, G., \& Clifford, M. N. (2010). Colonic metabolites of berry polyphenols: The missing link to biological activity? British Journal of Nutrition, 104(Suppl. 3), 48-66.

Xia, E. Q., Deng, G. F., Guo, Y. J., \& Li, H. B. (2010). Biological activities of polyphenols from grapes. International. Journal of Molecular Sciences, 11, 622-646.

Yasui, Y., Kim, M., Oyama, T., \& Tanaka, T. (2009). Colorectal carcinogenesis and suppression of tumor development by inhibition of enzymes and molecular targets. Current Enzyme Inhibition, 5, 1-26. 